TITLE:

\title{
Effects of RGDS sequence genetically interfused in the silk fibroin light chain protein on chondrocyte adhesion and cartilage synthesis.
}

\section{AUTHOR(S):}

Kambe, Yusuke; Yamamoto, Koji; Kojima, Katsura; Tamada, Yasushi; Tomita, Naohide

\section{CITATION:}

Kambe, Yusuke ...[et al]. Effects of RGDS sequence genetically interfused in the silk fibroin light chain protein on chondrocyte adhesion and cartilage synthesis.. Biomaterials 2010, 31(29): 7503-7511

\section{ISSUE DATE:}

2010-10

URL:

http://hdl.handle.net/2433/128943

\section{RIGHT:}

(c) 2010 Elsevier Ltd; この論文は出版社版でありません。引用の際には 出版社版をご確認ご利用ください。; This is not the published version. Please cite only the published version. 
Title

Effects of RGDS sequence genetically interfused in the silk fibroin light chain protein on chondrocyte adhesion and cartilage synthesis

\section{Authors}

Yusuke Kambe $^{1}$, Koji Yamamoto ${ }^{1}$, Katsura Kojima ${ }^{2}$, Yasushi Tamada ${ }^{2}$ and Naohide Tomita ${ }^{1, *}$

\section{Affiliations}

${ }^{1}$ Department of Mechanical Engineering, Graduate School of Engineering, Kyoto University, Yoshida-Honmachi, Sakyo-ku, Kyoto 606-8501, Japan

${ }^{2}$ National Institute of Agrobiological Sciences, 1-2 Owashi, Tsukuba, Ibaraki 305-8634, Japan

*Corresponding author

Naohide Tomita

Department of Mechanical Engineering, Graduate School of Engineering, Kyoto University, Yoshida-Honmachi, Sakyo-ku, Kyoto 606-8501, Japan

Tel/Fax: +81-75-753-9201

E-mail: ntomita@iic.kyoto-u.ac.jp

\section{Abstract}

Initial chondrocyte-silk fibroin interactions are implicated in chondrogenesis when using fibroin as a scaffold for chondrocytes. Here, we focused on integrin-mediated cell-scaffold adhesion and prepared cell adhesive fibroin in which a tandem repeat of the Arg-Gly-Asp-Ser (RGDS) sequence was genetically interfused in the fibroin light chain (L-chain) (L-RGDSx2 fibroin). We investigated the effects of the sequence on chondrocyte adhesion and cartilage synthesis, in comparison to the effects of fibronectin. As the physicochemical surface properties (e.g., wettability and $\zeta$ potential) of the fibroin substrate were not affected by the modification, specific cell adhesion to the RGDS predominately changed the chondrocyte adhesive state. This suggestion was also supported by the competitive inhibition of chondrocyte attachment to the L-RGDSx2 fibroin substrate with soluble RGD peptides in the medium. Unlike fibronectin, the expression of RGDS in the fibroin L-chain had no effect on 
chondrocyte spreading area but enhanced mRNA expression levels of integrins $\alpha 5$ and $\beta 1$, and aggrecan at $12 \mathrm{hrs}$ after seeding. Although both the sequence and fibronectin increased cell adhesive force, chondrocytes grown on the fibroin substrate exhibited a peak in the force with time in culture. These results suggested that moderate chondrocyte adhesion to fibroin induced by the RGDS sequence was able to maintain the chondrogenic phenotype and, from the histology findings, the sequence could facilitate chondrogenesis.

\section{Key words}

Cell adhesion, Chondrocyte, Scaffold, Silk, RGD peptide

\section{Introduction}

A large number of cells with the chondrogenic phenotype are required for clinical cartilage regeneration. However, the cellular supply source is limited when autologous cartilage cells are used for tissue-engineered therapy. To resolve this problem, numerous biomaterials have been developed for tissue regeneration by applying chemical and/or genetic modification technologies, and focusing on modulation of cell adhesive activities [1-9]. Our previous studies have shown that chondrocytes cultured using a porous silk fibroin sponge can proliferate well and synthesize glycosaminoglycan (GAG) which consists of cartilage-specific extracellular matrices (ECMs) [10]. We have also reported the in vivo functions of a silk fibroin scaffold for cartilage repair in a rabbit model [11]. Subsequently, Yamamoto et al. focused on the initial cell adhesion to the fibroin scaffold and investigated the time-dependent adhesive states between a chondrocyte and a fibroin substrate by evaluating the cell detachment force [12]. They showed that the adhesive force per unit cell spreading area of chondrocytes grown on a fibroin substrate was at a maximum value between 6 and 9 hrs after seeding; this peak was not observed in chondrocytes grown on a glass substrate, where the cells were reported not to proliferate or synthesize cartilage matrices [13]. The results of these studies indicate that fibroin-induced cell adhesion is associated with the stability of the chondrogenic phenotype and cartilage ECM synthesis.

An Arg-Gly-Asp (RGD) amino acid sequence is the minimum unit of a cell adhesive activity domain in adherent proteins, such as fibronectin, fibrin, and vitronectin, which are all ligands of integrins [14-16]. In addition the sequence is generally used to modulate cell adhesion to artificial materials. In culture, simple addition of RGD peptides or adherent proteins to a scaffold tends to facilitate the dedifferentiation of chondrocytes [5,17]. However, artificially immobilized RGD in/on scaffold components applied with physical or chemical modifications was reported to have some stimulatory effects on the synthesis of GAG $[7,8]$. 
Tigli and Gumusderelioglu reported that murine chondrogenic ATDC5 cells cultured in a chitosan scaffold modified with RGD by covalent immobilization produced more GAG than cells grown in a control chitosan scaffold [7]. Jung et al. also reported that chondrocytes cultured in acrylic acid-grafted poly(L-lactic acid) scaffolds on which RGD peptides were chemically immobilized showed higher cellularity and increased accumulation of GAG than control cells [8].

In the present study, we have investigated the effect of changes in fibroin-induced cell adhesion on cartilage tissue formation using RGDS-transgenic fibroin, particularly focusing on the initial chondrocyte-material adhesion. Fibroin protein consists of a heavy chain (H-chain; molecular weight, $360 \mathrm{kDa}$ ) and a light chain (L-chain; molecular weight, $27 \mathrm{kDa}$ ), which are linked by a disulfide bond to form a heterodimer [18]. There have been several studies of genetic modification of the H-chain [19] or the L-chain [20-23]. As the L-chain protein can be cloned and modified more easily than the H-chain [19], a tandem repeat of Arg-Gly-Asp-Ser ((RGDS $)_{2}$ ) was successfully interfused by genetic engineering methods [24] and stably expressed in the fibroin L-chain (L-RGDSx2 fibroin). The initial adhesion of chondrocytes on the substrate was evaluated by measuring adhesive force and spreading area. Cell morphology was also observed by immunofluorescence staining of F-actin and vinculin, and relative messenger RNA (mRNA) expression levels were measured. Additionally, the performance of L-RGDSx2 fibroin sponge as a chondrocyte scaffold was evaluated histologically.

\section{Materials and Methods}

\subsection{Chondrocytes}

Chondrocytes were prepared as described previously [10-12]. Briefly, articular cartilage tissues were aseptically harvested from the proximal humerus, distal femur and proximal tibia of 4-week-old Japanese white rabbits (Oriental Bio Service Co., Ltd., Japan). Chondrocytes were isolated via enzymatic digestion. After obtaining a single-cell suspension, the cells were cultured to $80 \%$ confluence on a T-flask (Asahi Glass Co., Ltd., Japan) with Dulbecco's modified Eagle's Medium (DMEM) (Nacalai Tesque, Inc., Japan) containing $10 \%$ fetal bovine serum (FBS) (Nacalai Tesque, Inc.) and 1\% antibiotic mixture (10,000 units/ml penicillin, 10,000 $\mu \mathrm{g} / \mathrm{ml}$ streptomycin, and $25 \mu \mathrm{g} / \mathrm{ml}$ amphotericin B) (Nacalai Tesque, Inc.) at $37^{\circ} \mathrm{C}$ in a humidified atmosphere of $95 \%$ air and $5 \% \mathrm{CO}_{2}$ for 5 to 7 days. The medium was changed every 2 or 3 days.

\subsection{Plasmids}


The plasmids used for silkworm transgenesis in this study are being prepared for publication. Briefly, pLp-LcDNA-EGFP-L3'UTR and pBac(3xP3-DsRed2afm) constructs were prepared as described by Inoue et al. [21]. An RGDSRGDS-fused fibroin-L-chain construct, pLLL-2xRGDSen, was prepared as follows. Two oligonucleotides (2xRGDS-5: 5'-GAT CGT GGA GAC AGC CGT GGA GAC AGC TGA-3' and 2xRGDS-3en: 5'-AGC TTC AGC TGT CTC CAC GGC TGT CTC CAC-3') were mixed at $100 \mathrm{pmol} / \mu \mathrm{l}$ each in polymerase chain reaction (PCR) buffer (TAKARA BIO Inc., Japan) for $1 \mathrm{~min}$ at $95^{\circ} \mathrm{C}$, and then incubated overnight at room temperature (RT) to prepare an oligonucleotide cassette. Following this, the cassette was ligated with BamHI and HindIII digested pLp-LcDNA-EGFP-L3'UTR. The resultant plasmid was designated pLLL-2xRGDSen. To obtain a transfer plasmid, the expression cassette in pLLL-2xRGDSen was digested into two pieces with HindIII and EcoRV, and HindIII and BglII. Finally, the resulting fragment was cloned between $B g l \mathrm{II}$ and the blunt-ended AscI site of pBac(3xP3-DsRed2afm), and then designated pBac(3xP3-DsRed2afm)_LLL-2xRGDSen.

\subsection{Generation of transgenic silkworms}

The genetic modification of the Bombyx mori silkworm performed in the present study is being prepared for publication. Briefly, silkworm transgenesis was performed as described by Tamura et al. [24] with minor modifications. The plasmid pBac(3xP3-DsRed2afm)_LLL-2xRGDSen was injected into silkworm eggs along with a helper plasmid at 3 to $6 \mathrm{hrs}$ post-oviposition. Hatched larvae (G0) were reared and permitted to mate with each other. The resultant embryos (G1) were screened by using a fluorescent microscope (MZ16 FA, Leica Microsystems, Germany) for transgenic individuals with DsRed2 expression 6 to 7 days after oviposition. The transgenic silkworms were reared together and sib-mated for at least three generations, with sequential screening by the strong excitation of DsRed2 fluorescence in the adult eye.

\subsection{Preparation of plate substrates and chondrocyte seeding}

Protein substrates were prepared using wild-type fibroin (WTF), L-RGDSx2 fibroin (LRF) and fibronectin (FN), and non-coated glass was used as a control substrate (CON). Proteins were coated on the following glass plates (Matsunami Glass Ind., Ltd., Japan): ultra-thin glass plates $(45 \times 1.5 \mathrm{~mm}$; thickness, $30 \mu \mathrm{m}$; Young's modulus, $71.4 \mathrm{GPa})$ for evaluating cell adhesive force; cover glass plates $(18 \times 18 \mathrm{~mm}$; thickness, $0.15 \mathrm{~mm})$ for evaluating cell spreading area and for real-time PCR analysis; cover glass plates (diameter, $15 \mathrm{~mm}$; thickness, $0.15 \mathrm{~mm}$ ) for measuring contact angles and for immunofluorescence staining of actin and vinculin; and cover glass plates $(24 \times 60 \mathrm{~mm}$; thickness, $0.15 \mathrm{~mm})$ for measuring $\zeta$ potential. All plates were made of borosilicate glass of the same quality. 
Wild-type silk fibroin aqueous solution was prepared from degummed silk fibers of $B$. mori silkworm cocoons [10-12]. The L-RGDSx2 fibroin protein contained modified fibroin L-chains fused with the (RGDS) $)_{2}$ sequences at the amino-terminus. The L-RGDSx 2 fibroin was produced by transgenic silkworms generated as described in section 2.3. The L-RGDSx2 fibroin aqueous solution was prepared using the same technique as for the preparation of the wild-type fibroin aqueous solution. The concentration of both fibroin solutions was adjusted to $1.0 \%$ (wt/vol) for all coating treatments, as described previously [12]. Fibroin-coated glass plates were extensively washed with phosphate-buffered saline (PBS) (Nacalai Tesque, Inc.) before use. Fibronectin substrates were prepared with ProNectin ${ }^{\circledR}$ F (Sanyo Chemical Industries, Ltd., Japan) according to the manufacturer's instructions. Briefly, a stock solution was diluted to $10 \mu \mathrm{g} / \mathrm{ml}$ in PBS, and glass plates were soaked in the diluted solution for 5 mins at RT. After removing the coating solution, the glass plates were washed twice with PBS.

After expansion in culture, chondrocytes were removed from the flask by mixing with $0.25 \%$ trypsin-EDTA (Nacalai Tesque, Inc.) and washed twice with PBS. The cells were seeded onto each substrate at $2.0 \times 10^{4}$ cells $/ \mathrm{cm}^{2}$ and incubated with DMEM containing $10 \%$ FBS and $1 \%$ antibiotic mixture at $37^{\circ} \mathrm{C}$ in a humidified atmosphere of $95 \%$ air and $5 \% \mathrm{CO}_{2}$.

\subsection{Preparation of fibroin sponges and chondrocyte seeding}

WTF sponges were prepared as described previously [10,11,25]. Briefly, a $4.0 \%$ (wt/vol) WTF solution was prepared from silkworm cocoons. After the addition of a water-soluble organic solvent ( $1 \%$ dimethyl sulfoxide), the fibroin protein solution was frozen for $24 \mathrm{hrs}$ to form a sponge-like material by phase separation. LRF sponges were prepared from transgenic silkworm cocoons using the same protocol for preparing WTF sponges. The sponges had a porous structure with a mean pore diameter of about $80 \mu \mathrm{m}$. They were sterilized in an autoclave before use.

After expansion, chondrocytes were trypsinized and rinsed twice with PBS. The cells were seeded onto the fibroin scaffold (diameter, $8 \mathrm{~mm}$; thickness, $1 \mathrm{~mm}$ ) at a concentration of $5.0 \times$ $10^{5}$ cells/scaffold and cultured with DMEM containing $10 \%$ FBS, $1 \%$ antibiotic mixture, and $0.2 \mathrm{mM}$ ascorbic acid (A8960, Sigma-Aldrich Co., USA) at $37^{\circ} \mathrm{C}$ in a humidified atmosphere of $95 \%$ air and $5 \% \mathrm{CO}_{2}$. The medium was changed every 2 days.

\subsection{Scanning electron microscopy (SEM)}

Scanning electron microscopy (SEM) was used to observe the effect of the genetic modification on the topology of fibroin films and fibroin sponges. In preparation of the fibroin-coated glass plates, the plates were washed extensively with ultrapure water and dried in a clean bench. Immediately following this, they were sputter-coated with gold-palladium 
using an E-1010 instrument (Hitachi Ltd., Japan) and imaged by a scanning electron microscope (S-2380N, Hitachi Ltd.). Three different specimens of each fibroin film were used for the observation.

In preparation of the fibroin sponges, the sponges were washed twice with PBS for 10 mins and dehydrated in a graded ethanol series $(70 \%, 80 \%, 90 \%, 95 \%$, and $100 \%$ ethanol for 5, 5, 10, 10, and 10 mins, respectively). The remaining ethanol was replaced by incubating twice in tert-butyl alcohol for 10 mins. Specimens were then frozen on dry ice and dried using an ES-2030 instrument (Hitachi Ltd.). Following this, the freeze-dried fibroin sponges were sputter-coated with gold-palladium and imaged as described above. Three different samples of each fibroin sponge were used for the observation.

\subsection{Chondrocyte attachment to substrates and competitive inhibition with soluble RGD peptides}

The influence of different substrates on the attachment of chondrocytes was observed using a phase-contrast microscope (IX-71, Olympus Corp., Japan). At 24 hrs after seeding, the cells on each substrate were washed twice with PBS and fixed with $4 \%$ paraformaldehyde (PFA) solution for 15 mins at RT. After removing the solution, a constant amount (1 ml) of PBS was added and then the attaching cells were photographed. Chondrocytes were incubated on LRF substrates with serum-free medium containing different concentrations $(0,0.1,1$, and 10 $\mu \mathrm{g} / \mathrm{ml}$ ) of Gly-Arg-Gly-Asp-Ser-Pro (GRGDSP) peptides (SP001, TAKARA BIO Inc.). After incubation for $24 \mathrm{hrs}$, the cells were photographed, as described above. Experiments were repeated at least three times, and similar results were noted each time.

\subsection{Measurement of surface properties of substrates}

The surface properties of the substrates as represented by contact angle and $\zeta$ potential were evaluated immediately after the samples were extensively washed with ultrapure water and dried in a clean bench. Static water contact angles on the substrates were determined by the sessile drop method using CA-Z2 (Kyowa Interface Science Co., Ltd., Japan) at RT. A droplet $(4 \mu \mathrm{l})$ of ultrapure water was placed on the surface of a sample and the contact angle was measured. This procedure was repeated three times at different areas on the same surface, and the contact angle of the sample was determined as the mean value of the three measurements. Three different samples in each group were used to determine the contact angle of the group.

The $\zeta$ potential of each substrate was measured using an ELS-7000AS instrument with the cell unit for flat plate samples (Otsuka Electronics Co., Ltd., Japan) in $10 \mathrm{mM} \mathrm{NaCl}$ at $25^{\circ} \mathrm{C}$. The $\mathrm{pH}$ of the streaming solution was adjusted to 7.4 with $\mathrm{HCl}$ and $\mathrm{NaOH}$ aqueous solutions. 
Three different samples in each group were used to determine the $\zeta$ potential of the group.

\subsection{Apparatus and procedure for measuring adhesive force}

Adhesive force was measured as described previously [12]. Briefly, after incubation for 3, 6, 9, 12, or $24 \mathrm{hrs,}$ an ultra-thin glass plate seeded with chondrocytes was fixed to the holder and completely submerged into a chamber filled with L-15 medium (Invitrogen Corp., USA; containing $10 \% \mathrm{FBS}$ and $1 \%$ antibiotic mixture) pre-warmed to $37^{\circ} \mathrm{C}$. The plate acted as a cantilever, and a micropipette aspirator was used to hold a single cell adhering to the substrate. The cell was detached by pulling the micropipette at a constant rate of $5 \mu \mathrm{m} / \mathrm{s}$ using a motorized single-axis stage (KS101-20HD, Suruga Seiki Co., Ltd., Japan). In this experiment, adhesive force was calculated as the maximum deflection of the glass plate observed through a video microscope (DG-2, Scalar Co., Japan) connected to a time-lapse video capture board (GV-MVP/RX3, I-O Data Device Inc., Japan). Force $(F)$ is given by $F=3 I E \omega L^{-3}$, where $E$ is Young's modulus, $I$ is the moment of inertia of the area, $L$ is the length from the fixed edge of a leaf spring to a cell, and $\omega$ is the maximum deflection at $L$, which can vary in accordance with the attachment position of a micropipette.

This measurement was performed at RT. The maximum deflection $\omega$ of the leaf spring was determined by counting the pixels using ImageJ (National Institute of Health, USA) from the photographs taken during the detaching process. The number of measurements of adhesive force in each group at 3, 6, 9, 12, and 24 hrs after seeding was as follows: WTF group, $\mathrm{n}=17$, 10, 13, 24, and 19; LRF group, $\mathrm{n}=13,13,7,9$, and 16; CON group, $\mathrm{n}=26,31,39,40$, and 27 ; and $\mathrm{FN}$ group, $\mathrm{n}=24,27,31,38$, and 36 , respectively.

\subsection{Measurement of cell spreading area}

Chondrocytes were seeded onto four different substrates as described above. After incubation for $3,6,9,12$, or $24 \mathrm{hrs}$, the cells were washed twice with PBS and then fixed with $4 \%$ PFA solution for 15 mins at $37^{\circ} \mathrm{C}$. The cells were then stained with $1 \%(\mathrm{wt} / \mathrm{vol})$ Coomassie Brilliant Blue solution for 2 mins and washed twice with PBS. Digital images of the cells were acquired by microscopy. Cell spreading area was measured by analyzing the binarized images using ImageJ. The Coomassie Brilliant Blue solution was prepared by dissolving Coomassie ${ }^{\circledR}$ Brilliant Blue R 250 (Sigma-Aldrich Co.) powder in an aqueous solution containing $50 \%$ methanol and $1 \%$ of acetic acid. The number of measurements of spreading area in each group at 3, 6, 9, 12, and $24 \mathrm{hrs}$ after seeding was as follows: WTF group, $\mathrm{n}=24,29,33,23$, and 28; LRF group, $\mathrm{n}=35,30,27,27$, and 27; CON group, $\mathrm{n}=29$, $34,44,38$, and 49; and FN group, $\mathrm{n}=44,44,37,58$, and 43, respectively.

\subsection{Immunofluorescence staining of F-actin and vinculin}


After incubation for 6, 12, or 24 hrs, F-actin, vinculin, and the nucleus of chondrocytes grown on the substrates were stained as described previously [12]. Briefly, after fixing with 4\% PFA solution, the cells were permeabilized in 0.1\% Triton X-100 (9690T, Research Organics, Inc., USA), and then incubated in fresh blocking solution prepared with $1 \%$ (wt/vol) bovine serum albumin (82-045-1, Serological Corp., USA). Rhodamine phalloidin (R415, Molecular Probes, USA), anti-vinculin (MAB3574, Chemicon International, Inc., USA) as a primary antibody and fluorescein isothiocyanate-conjugated secondary antibody (AP124F, Chemicon International, Inc.), and 4',6-diamidino-2-phenylindole (S7113, Chemicon International, Inc.) were used to stain F-actin, vinculin, and the nucleus, respectively. Stained chondrocytes were observed with an inverted fluorescence microscope (IX-71 and BH2-RFL-T3, Olympus Corp.).

\subsection{Real-time PCR analysis}

After incubation for 6, 12, or $24 \mathrm{hrs,} \mathrm{chondrocytes} \mathrm{cultured} \mathrm{on} \mathrm{each} \mathrm{substrate} \mathrm{were} \mathrm{washed}$ twice with ice-cold PBS and then removed from the substrate by scraping with a cell scraper (Asahi Glass Co., Ltd.) on ice. mRNA was extracted from the cells using the mRNA Capture kit (Roche Diagnostics, Germany), and then cDNA was synthesized by reverse-transcription PCR using the Transcriptor First Strand cDNA Synthesis kit (Roche Diagnostics) and thermal cycler (PC-320, Astec Co., Ltd., Japan). The synthesized cDNA from each group was stored at $-20^{\circ} \mathrm{C}$ until use.

Real-time PCR was performed using the LightCycler ${ }^{\circledR}$ FastStart DNA Master ${ }^{\text {PLUS }}$ SYBR Green I (Roche Diagnostics), and SYBR Green PCR amplification and real-time fluorescence detection were performed using the LightCycler $^{\circledR}$ ST300 (Roche Diagnostics). Primers (Sigma-Aldrich Co.) were designed for glyceraldehyde-3-phosphate dehydrogenase (GAPDH) as the house-keeping gene, integrins $\alpha 5$ and $\beta 1$, aggrecan, and collagen type II $\alpha \mathrm{I}$ chain. Primer sequences for the rabbit GAPDH gene (PudMed ID\#: L23961) were as follows: forward primer 5'-TCA CCA TCT TCC AGG AGC GA-3'; reverse primer 5'-CAC AAT GCC GAA GTG GTC GT-3' [26]. Primer sequences for the rabbit integrin $\alpha 5$ gene (PudMed ID\#: S77513) were as follows: forward primer 5'-GGC AGC TAT GGC GTC CCA CTG TGG-3'; reverse primer 5'-GGC ATC AGA GGT GGC TGG AGG CTT-3' [27]. Primer sequences for the rabbit integrin $\beta 1$ gene (PudMed ID\#: S77516) were as follows: forward primer 5'-GTG GTT GCT GGA ATT GTT CTT ATT-3'; reverse primer 5'-TTT TCC CTC ATA CTT CGG ATT GAC-3' [27]. Primer sequences for the rabbit aggrecan gene (PudMed ID\#: L38480) were as follows: forward primer 5'-CTA CGA CGC CAT CTG CTA CA-3'; reverse primer 5'-CCT CTT CAG TCC CGT TCT-3'. Primer sequences for the rabbit collagen type II $\alpha$ I chain gene (PudMed ID\#: S83370) were as follows: forward primer 5'-GCA CCC ATG GAC ATT GGA GGG-3'; reverse primer 5'-GAC ACG GAG TAG CAC CAT CG-3' [28]. In each 
group at each time point, $\mathrm{n}=4$ for mRNA expression levels, except for the levels of aggrecan in the WTF and LRF groups at $6 \mathrm{hrs}$ after seeding $(\mathrm{n}=3)$.

\subsection{Histological evaluation of regenerated cartilage}

After culturing for 3 or 7 days, regenerated cartilage organized in/on WTF or LRF sponges was fixed with $20 \%$ neutral buffered formalin and incubated for more than $24 \mathrm{hrs}$ at $4{ }^{\circ} \mathrm{C}$. Samples were embedded in paraffin and cut into $7-\mu$ m-thick sections. Specimens were sectioned parallel to the length of the scaffold, and stained for safranin $\mathrm{O}$ and immunostained for collagen type I (F-56, DAIICHI FINE CHEMICAL Co., Ltd., Japan) and type II (F-57, DAIICHI FINE CHEMICAL Co., Ltd.). Photographs of stained samples were taken under a microscope. From each group, three samples were prepared for the staining, and for repeatability, culture experiments were performed in triplicate.

\subsection{Statistical analysis}

Quantitative data were analyzed between WTF and LRF groups and between CON and FN groups using two-sided Student's t-test. A value of $p<0.05$ was considered significant. In all figures, asterisks indicate statistical significance between WTF and LRF groups, and sharp symbols indicate differences between CON and FN groups.

\section{Results}

\subsection{Observations of fibroin films by SEM and chondrocyte attachment}

The representative scanning electron micrographs of the WTF and LRF films are shown in Fig. 1A. Both surfaces were almost uniform, and no marked topological differences between the WTF and LRF substrates were observed.

Chondrocytes cultured on each substrate for $24 \mathrm{hrs}$ are shown in Fig. 1B. The number of cells that attached to the FN and LRF substrates appeared to be higher than the number of cells that attached to the CON and WTF substrates. However, as shown in Fig. 1C, chondrocyte adhesion to an LRF substrate was inhibited dose-dependently by GRGDSP peptide in the medium.

\subsection{Surface properties of substrates}

Surface properties such as contact angle and $\zeta$ potential of each substrate are shown in 
Table 1. There were no significant differences in either contact angle or $\zeta$ potential between the WTF and LRF groups or between the CON and FN groups.

\subsection{Cell adhesive force}

The time-dependent changes in adhesive force of chondrocytes grown on each substrate are shown in Fig. 2. The LRF and FN groups tended to show higher adhesive force than the WTF and CON groups, respectively. However, during $12 \mathrm{hrs}$ after seeding, the LRF group demonstrated more force than the WTF group, while the FN group showed more force than the CON group after $12 \mathrm{~h}$. The adhesive force of the LRF group was significantly higher than that of the WTF group at 3, 9, and 12 hrs after seeding, but significantly lower at 24 hrs. The FN group showed significantly higher adhesive force than the CON group only at 12 hrs after seeding. In the WTF and LRF groups, the adhesive force did not change monotonically as in the CON and FN groups, but instead reached a maximum value within $12 \mathrm{hrs}$ after seeding.

\subsection{Cell spreading area}

The cell spreading area as a function of culture time is shown in Fig. 3. No significant differences were found between the WTF and LRF groups; both groups tended to show similar temporal changes in spreading area. On the other hand, chondrocytes grown on the FN substrate tended spread to a larger area than those grown on the CON substrate, and these effects were significant at 6 and $24 \mathrm{hrs}$ after seeding.

\subsection{Cell morphology}

The representative photographs of F-actin and vinculin staining for each substrate at 6 and $12 \mathrm{hrs}$ after seeding are shown in Fig. 4. At 6 hrs after seeding, immature actin fibers and dot-like vinculin were sporadically present in chondrocytes grown on both the WTF and LRF substrates. At $12 \mathrm{hrs}$, this cell morphology was not observed but polymerized actin fibers were formed, and dash-like vinculin was observed at the cell processes in the LRF group. Finally, at $24 \mathrm{hrs}$ after seeding, the latter F-actin structure and vinculin distributions were observed in both the WTF and LRF groups (data not shown). As described above, temporal morphology changes were shown in chondrocytes cultured on the two fibroin substrates. In contrast, polymerized actin fibers and dash-like vinculin were constantly observed in the CON and FN groups.

\section{6. mRNA expression levels}


Figure 5 shows the transitions of the mRNA expression levels of integrins $\alpha 5$ and $\beta 1$, aggrecan, and collagen type II at 6,12 , and $24 \mathrm{hrs}$ after seeding normalized with respect to the expression level at baseline $(0 \mathrm{hr})$. The aggrecan expression in the WTF and LRF groups showed a peak within $24 \mathrm{hrs}$ after seeding, whereas the aggrecan expression in the CON and FN groups only changed slightly with time. The LRF group exhibited higher expression of aggrecan and integrins $\alpha 5$ and $\beta 1$ at $12 \mathrm{hrs,}$, whereas there were no significant differences in the mRNA expression levels between the $\mathrm{CON}$ and $\mathrm{FN}$ groups.

\subsection{SEM observation of fibroin sponges and histology findings}

Figure 6A shows the representative scanning electron micrographs for the WTF and LRF sponges. Both sponges appeared to be similar in their morphology, possessing similar pore size and pore size distribution.

Photographs of stained regenerated cartilage synthesized on the WTF and LRF scaffolds are shown in Fig. 6B. Cartilage-like tissues were better formed in the LRF group than in the WTF group after 3 days in culture. Furthermore, after culturing for 7 days, two- to three-fold thicker matrices were formed at the surface of the LRF scaffold compared to the WTF scaffold. The expression of collagen type I was not detected in both groups.

\section{Discussion}

It is generally thought that introduction of RGD peptides into a substrate facilitates integrin-mediated cell attachment. In the present study, this theory was confirmed by the fact that the FN and LRF substrates had a higher cell-adhesive activity than the CON and WTF substrates, respectively, as shown in Fig. 1B. However, it is widely accepted that in addition to integrin-mediated biologic adhesion, surface properties of a material (e.g., wettability and charge) greatly affect cell-substrate adhesion [29-34]. In the present study, there were no marked differences in contact angle or $\zeta$ potential between the WTF and LRF substrates or between the CON and FN substrates, suggesting that the physicochemical properties of the substrates have little impact on levels of chondrocyte adhesion.

Integrins appear to be the major cell surface receptors by which a cell can adhere mechanically to ECMs, and chondrocytes have been shown to express several members of the integrin family [35]. Competitive inhibition of chondrocyte attachment to an LRF substrate with soluble GRGDSP peptides indicated that integrin receptors on a suspending chondrocyte were blocked by the peptides and could not bind to ligands on the surface of a substrate. These results suggest that differences in cell-adhesive activity between the WTF and LRF substrates were caused by ligands that did not exist on the WTF substrate, that is, RGDS sequences expressed in the fibroin L-chain proteins. Therefore, RGDS in the L-chain likely 
affected adhesion, suggesting that biologic rather than physicochemical adhesion was predominant in the present experiments.

Generally, by the enhancement of chondrocyte adhesion to their scaffold, chondrocytes tend to lose their chondrogenic phenotype $[5,6,17]$, and mechanical adhesion is affected to increase the cell adhesive force [36]. The latter effect was seen with both RGDS expressed in the fibroin L-chain and fibronectin coated on a glass substrate. The mRNA expression of aggrecan, which encodes cartilage-specific proteoglycan, peaked at a similar level in the WTF and LRF groups, although its expression was delayed in the latter. In addition, chondrocytes grown on L-RGDSx2 fibroin sponges could better synthesize cartilage in ECMs than those cultured on WTF sponges during the early culture period. These results suggest that RGDS genetically interfused in the L-chain can facilitate cell adhesive strength, without down-regulating the chondrocyte-specific phenotype.

Chondrocytes grown on the WTF and LRF substrates exhibited a maximum adhesive force within 12 hrs after seeding, but both the CON and FN groups showed only a monotonic increase. Others have also shown monotonous enhancement of adhesive force in cells cultured on glass, fibronectin, or a culture dish, although the detaching process and direction of force measurement were different [36-38]. These results could indicate that the specific changes in adhesive force of the WTF and LRF groups were predominantly affected by the fibroin, and that the RGDS sequences in the L-chain had an influence on the expression period and the peak value. However, the RGDS appeared to have no effect on cell spreading, unlike the enhancement effect of fibronectin coated on a glass substrate and RGD peptides fixed on a substrate as seen in both the present and previous studies [1,2].

Considering that proteoglycan has been reported to inhibit and regulate integrin-mediated cell-material adhesion [39,40], the specific gene expression of integrins $\alpha 5$ and $\beta 1$, which recognize an RGD sequence in ECMs [14-16,35], and aggrecan in the LRF group could be related to the adhesive force. Furthermore, higher gene expression of integrins in the LRF group might affect the earlier changes in actin polymerizations and distributions of focal adhesion points. This is supported by observations that integrins bind to the cytoskeleton (to a polymerized actin called F-actin), mediated by anchor molecules such as vinculin, talin, and $\alpha$-actinin, and clusters to construct focal adhesions [41-43]. Although the development of F-actin and the expression of focal adhesion points are important factors for adhesive force [12,36-38], there appears to be no relations between them in chondrocytes grown on the two fibroin substrates.

The results of the present study suggest that RGDS amino acids genetically interfused in the L-chain protein of the silk fibroin facilitate cartilage tissue formation as shown in Fig. $6 \mathrm{~B}$ but, in contrast to fibronectin, do not induce spreading and dedifferentiation of chondrocytes. Hsu et al. suspected that the effect of RGD could be substrate (scaffold)-dependent [5]. Jung et al. suggested that an optimal concentration of RGD might vary in different cell or substrate types and help to form cartilaginous tissues, but very strong adhesion is likely to suppress 
matrix production [8]. The results of adhesion of a single chondrocyte grown on an LRF substrate suggest that adhesion between a chondrocyte and an L-RGDSx 2 fibroin substrate, unlike fibronectin, was not excessively strong; instead, moderate adhesion was sufficient to maintain the chondrogenic phenotype. In particular, the specific enhancement effect on peak adhesive force might influence the organization of cartilage tissues because a peak was not observed in the force of chondrocytes cultured on glass or fibronectin substrates, on which chondrocytes cannot synthesize hyaline-like cartilage tissues [13,17]. Although it is difficult to precisely explain the mechanisms involved in the specific behavior of initial adhesion and chondrogenesis from the results of the present study alone, changes in adhesive force are crucial via the deformation of cell membranes, the distribution pattern of focal contacts, and the effect of substances synthesized by chondrocytes, as described previously by Yamamoto et al. [12]. These factors can affect the signal cascade of phenotypic expression and/or production of ECMs $[44,45]$. Hence, considering that there was no significant difference in the number of chondrocytes initially trapped on/in WTF or LRF scaffolds [46], early integrin-mediated signal changes induced by the adhesion to LRF within 24 hrs (i.e., the increase and/or delay of integrin mRNA expressions as shown in Fig. 5) might stimulate chondrogenesis. Another possibility is that the adhesive force of chondrocytes affected their migration and aggregation, which resulted in the formation of cartilaginous tissues. This suggestion is supported by the findings that cell adhesive strength is related to cell motility $[47,48]$ and that chondrocyte movement and distribution in a fibroin sponge play a central role in the production of cartilage-specific matrices (unpublished observations).

A fibroin $\mathrm{H}$-chain protein is reported to be composed of a crystalline region linked with an amorphous region [49], whereas it is assumed that there are no crystal structures in an L-chain $[18,50]$ (where the RGDS sequences are fused in the present study). On the other hand, ProNectin ${ }^{\circledR} \mathrm{F}$, used as fibronectin in this study, is reported to be composed of the crystallized sequence containing (Gly-Ala-Gly-Ala-Gly-Ser $)_{9}\left((\text { GAGAGS })_{9}\right)$ from fibroin H-chain, which forms a $\beta$-sheet structure where a 10 amino acid sequence from fibronectin containing RGDS is presented in turn [51,52]. Additionally, cell-adhesive activity of an RGD sequence can be affected by its conformation [53,54]. Therefore, the specific effects of RGDS in the fibroin L-chain might be ascribable to the activity of the amino acids and the conformational effects of the sequences. However, to confirm these theories it is necessary to investigate these factors further in future studies.

\section{Conclusions}

The results of the present study have demonstrated that chondrocytes grown on the L-RGDSx2 fibroin substrate show similar spreading area but higher mRNA expression levels 
of integrins $\alpha 5$ and $\beta 1$ at $12 \mathrm{hrs}$ after seeding compared to the cells on the wild-type fibroin substrate. Further, the specific enhancement of the peak adhesive force has been exhibited within $12 \mathrm{hrs}$. These changes were not shown between chondrocytes culture on glass and fibronectin substrates. The study also suggests that the sequence in the L-chain can facilitate the synthesis of cartilage tissue.

\section{Acknowledgements}

The authors would like to thank Assistant Prof. Masaya Yamamoto and Prof. Yasuhiko Tabata from the Department of Biomaterials, Institute for Frontier Medical Sciences, Kyoto University for helping to measure the $\zeta$ potential of the substrates; Mr. Takahisa Matsushita and Ms. Kumiko Kogishi from the Technical Department, Institute for Frontier Medical Sciences, Kyoto University for helping with histological staining of regenerated cartilage; and Kyoto University-Venture Business Laboratory for use of their experimental laboratory. This study was supported in part by Integrated Research Project for Plant, Insect and Animal Using Genome Technology (MAFF) and Grant-in-Aid for Creative Scientific Research (Japan Science and Technology Agency).

\section{References}

[1] Ohno S, Noshiro M, Makihira S, Kawamoto T, Shen M, Yan, W, et al. RGD-CAP ( $\beta$ ig-h3) enhances the spreading of chondrocytes and fibroblasts via integrin $\alpha_{1} \beta_{1}$. Biochim Biophys Acta 1999;1451:196-205.

[2] Jeschke B, Meyer J, Jonczyk A, Kessler H, Adamietz P, Meenen NM, et al. RGD-peptides for tissue engineering of articular cartilage. Biomaterials 2002;23:3455-63.

[3] Genes NG, Rowley JA, Mooney DJ, Bonassar LJ. Effect of substrate mechanics on chondrocyte adhesion to modified alginate surfaces. Arch Biochem Biophys 2004;422:161-7.

[4] Meinel L, Hofmann S, Karageorgiou V, Zichner L, Langer R, Kaplan D, et al. Engineering cartilage-like tissue using human mesenchymal stem cells and silk protein scaffolds. Biotechnol Bioeng 2004;88:379-91.

[5] Hsu SH, Chang SH, Yen HJ, Whu SW, Tsai CL, Chen DC. Evaluation of biodegradable polyesters modified by type II collagen and Arg-Gly-Asp as tissue engineering scaffolding materials for cartilage regeneration. Artif Organs 2006;30:42-55.

[6] Connelly JT, Garcia AJ, Levenston ME. Inhibition of in vitro chondrogenesis in RGD-modified three-dimensional alginate gels. Biomaterials 2007;28:1071-83.

[7] Tigli RS, Gumusderelioglu M. Evaluation of RGD- or EGF-immobilized chitosan scaffolds for chondrogenic activity. Int J Biol Macromol 2008;43:121-8. 
[8] Jung HJ, Park K, Kim JJ, Lee JH, Han KO, Han DK. Effect of RGD-immobilized dual-pore poly(L-lactic acid) scaffolds on chondrocyte proliferation and extracellular matrix production. Artif Organs 2008;32:981-9.

[9] Meinel AJ, Kubow KE, Klotzsch E, Garcia-Fuentes M, Smith ML, Vogel V, et al. Optimization strategies for electrospun silk fibroin tissue engineering scaffolds. Biomaterials 2009;30:3058-67.

[10] Aoki H, Tomita N, Morita Y, Hattori K, Harada Y, Sonobe M, et al. Culture of chondrocytes in fibroin-hydrogel sponge. Biomed Mater Eng 2003;13:309-16.

[11] Chueh S, Tomita N, Yamamoto K, Harada Y, Nakajima M, Terao T, et al. Transplantation of allogeneic chondrocytes cultured in fibroin sponge and stirring chamber to promote cartilage regeneration. Tissue Eng 2007;13:483-92.

[12] Yamamoto K, Tomita N, Fukuda Y, Suzuki S, Igarashi N, Suguro T, et al. Time-dependent changes in adhesive force between chondrocytes and silk fibroin substrate. Biomaterials 2007;28:1838-46.

[13] Park GE, Pattison MA, Park K, Webster TJ. Accelerated chondrocyte function on $\mathrm{NaOH}$-treated PLGA scaffolds. Biomaterials 2005;26:3075-82.

[14] Rouslahti E, Pierschbacher MD. Arg-Gly-Asp: a versatile cell recognition signal. Cell 1986;44:517-8.

[15] Rouslahti E, Pierschbacher MD. New perspectives in cell adhesion: RGD and integrins. Science 1987;23:491-7.

[16] Hynes RO. Integrins: versatility, modulation, and signaling in cell adhesion. Cell 1992;69:11-25.

[17] Brodkin KR, Garcia AJ, Levenston ME. Chondrocyte phenotypes on different extracellular matrix monolayers. Biomaterials 2004;25:5929-38.

[18] Tanaka K, Kajiyama N, Ishikura K, Waga S, Kikuchi A, Ohtomo K, et al. Determination of the site of disulfide linkage between heavy and light chains of silk fibroin produced by Bombyx mori. Biochim Biophys Acta 1999;1432:92-103.

[19] Kojima K, Kuwana Y, Sezutsu H, Kobayashi I, Uchino K, Tamura T, et al. A new method for the modification of fibroin heavy chain protein in the transgenic silkworm. Biosci Biotechnol Biochem 2007;71:2943-51.

[20] Tomita M, Munetsuna H, Sato T, Adachi T, Hino R, Hayashi M, et al. Transgenic silkworms produced recombinant human type III procollagen in cocoons. Nat Biotechnol 2003;21:52-6.

[21] Inoue S, Kanda $T$, Imamura $M$, Quan GX, Kojima K, Tanaka H, et al. A fibroin secretion-deficient silkworm mutant, $N d-s^{D}$, provides an efficient system for producing recombinant proteins. Insect Biochem Mol Biol 2005;35:51-9.

[22] Adachi T, Tomita M, Shimizu K, Ogawa S, Yoshizato K. Generation of hybrid transgenic silkworms that express Bombyx mori prolyl-hydroxylase $\alpha$-subunits and human collagens in posterior silk glands: production of cocoons that contained collagens with hydroxylated 
proline residues. J Biotechnol 2006;126:205-19.

[23] Yanagisawa S, Zhu Z, Kobayashi I, Uchino K, Tamada Y, Tamura T, et al. Improving cell-adhesive properties of recombinant Bombyx mori silk by incorporation of collagen of fibronectin derived peptides produced by transgenic silkworms. Biomacromolecules 2007;8:3487-92.

[24] Tamura T, Thibert C, Royer C, Kanda T, Abraham E, Kamba M, et al. Germline transformation of the silkworm Bombyx mori L. using a piggyBac transposon-derived vector. Nat Biotechnol 2000;18:81-4.

[25] Tamada Y. New process to form a silk fibroin porous 3-D structure. Biomacromolecules 2005;5:217-28.

[26] Majima T, Marchuk LL, Shrive NG, Frank CB, Hart DA. In-vitro cyclic loading of an immobilized and mobilized ligament autograft selectively inhibits mRNA levels for collagenase (MMP-1). J Orthop Sci 2000;5:503-10.

[27] Ohashi H, Maeda T, Mishima H, Otori T, Nishida T, Sekiguchi K. Up-regulation of integrin $\alpha 5 \beta 1$ expression by interleukin-6 in rabbit corneal epithelial cells. Exp Cell Res 1995;218:418-23.

[28] Huang CY, Hangar KL, Frost LE, Sun Y, Cheung HS. Effects of cyclic compressive loading on chondrogenesis of rabbit bone-marrow derived mesenchymal stem cells. Stem Cells 2004;22:313-23.

[29] Van Wachem PB, Hogt AH, Beugeling T, Feijen J, Bantjes A, Detmers JP, et al. Adhesion of cultured human endothelial cells onto methacrylate polymers with varying surface wettability and charge. Biomaterials 1987;8:323-8.

[30] Kishida A, Iwata H, Tamada Y, Ikada Y. Cell behaviour on polymer surfaces grafted with non-ionic and ionic monomers. Biomaterials 1991;12:786-92.

[31] Tamada Y, Ikada Y. Cell adhesion to plasma-treated polymer surfaces. Polymer 1993;34:2208-12.

[32] Lee JH, Lee JW, Khang G, Lee HB. Interaction of cells on chargeable functional group gradient surfaces. Biomaterials 1997;18:351-8.

[33] Arima $\mathrm{Y}$, Iwata $\mathrm{H}$. Effect of wettability and surface functional groups on protein adsorption and cell adhesion using well-defined mixed self-assembled monolayers. Biomaterials 2007;28:3074-82.

[34] Arima Y, Iwata H. Effects of surface functional groups on protein adsorption and subsequent cell adhesion using self-assembled monolayers. J Mater Chem 2007;17:4079-87.

[35] Loeser RF. Chondrocyte integrin expression and function. Biorheology 2000;37:109-16.

[36] Athanasiou KA, Thoma BS, Lanctot DR, Shin D, Agrawal CM, LeBaron RG. Development of the cytodetachment technique to quantify mechanical adhesiveness of the single cell. Biomaterials 1999;20:2405-15.

[37] Hoben G, Huang W, Thoma BS, LeBaron RG, Athanasiou KA. Quantification of varying adhesion levels in chondrocytes using the cytodetacher. Ann Biomed Eng 2002;30:703-12. 
[38] Huang W, Anvari B, Torres JH, LeBaron RG, Athanasiou KA. Temporal effects of cell adhesion on mechanical characteristics of the single chondrocyte. J Orthop Res 2003;21:88-95.

[39] Yamagata M, Suzuki S, Akiyama SK, Yamada KM, Kimata K. Regulation of cell-substrate adhesion by proteoglycans immobilized on extracellular substrates. J Biol Chem 1989;264:8012-8.

[40] Imoto E, Kakuta S, Hori M, Yagami K, Nagumo M. Adhesion of a chondrocytic cell line (USAC) to fibronectin and its regulation by proteoglycan. J Oral Pathol Med 2002;31:35-44.

[41] Greenwood JA, Murphy-Ullrich JE. Signaling of de-adhesion in cellular regulation and motility. Microsc Res Tech 1998;43:420-32.

[42] Giancotti FG, Ruoslahti E. Integrin signaling. Science 1999;285:1028-32.

[43] Martin KH, Slack JK, Boerner SA, Martin CC, Parsons JT. Integrin connections map: to infinity and beyond. Science 2002;296:1652-3.

[44] Enomoto M, Leboy PS, Menko AS, Boettiger D. $\beta 1$ integrins mediate chondrocyte interaction with type I collagen, type II collagen, and fibronectin. Exp Cell Res 1993;205:276-85.

[45] Takagi M, Kitabayashi T, Koizumi S, Hirose H, Kondo S, Fujikawa M, et al. Correlation between cell morphology and aggrecan gene expression level during differentiation from mesenchymal stem cell to chondroytes. Biotechnol Lett 2008;30:1189-95.

[46] Kambe Y, Yamamoto K, Kojima K, Tamada Y, Tomita N. Adhesion of a single chondrocyte on RGDS-transgenic fibroin substrate and tissue formation (in Japanese). Jpn J Clin Biomech 2009;30:71-6.

[47] DiMilla PA, Barbee K, Lauffenburger DA. Mathematical model for the effects of adhesion and mechanics on cell migration speed. Biophys J 1991;60:15-37.

[48] DiMilla PA, Stone JA, Quinn JA, Albelda SM, Lauffenburger DA. Maximal migration of human smooth muscle cells on fibronectin and type IV collagen occurs at an intermediate attachment strength. J Cell Biol 1993;122:729-37.

[49] Mita K, Ichimura S, James TC. Highly repetitive structure and its organization of the silk fibroin gene. J Mol Evol 1994;38:583-92.

[50] Yamaguchi K, Kikuchi Y, Takagi T, Kikuchi A, Oyama F, Shimura K, et al. Primary structure of the silk fibroin light chain determined by cDNA sequencing and peptide analysis. J Mol Biol 1989;210:127-39.

[51] Cappello J, Crissman JW. The design and production of bioactive protein polymers for biomedical applications. Polymer Prepr 1990;31:193-4.

[52] Anderson JP, Cappello J, Martin DC. Morphology and primary crystal structure of a silk-like protein polymer synthesized by generically engineered $E$. Coli bacteria. Biopolymers 1994;34:1049-57.

[53] Maeda T, Oyama R, Ichihara-Tanaka K, Kimizuka F, Kato I, Titani K, et al. A novel cell adhesive protein engineered by insertion of the Arg-Gly-Asp-Ser tetrapeptide. J Biol Chem 
1989;264:15165-8.

[54] Hersel U, Dahmen C, Kessler H. RGD modified polymers: biomaterials for stimulated cell adhesion and beyond. Biomaterials 2003;24:4385-415. 
Table 1

Surface properties of each substrate as represented by contact angle and $\zeta$ potential.

\begin{tabular}{lll}
\hline Substrate & Contact angle $[\mathrm{deg}]$ & $\zeta$ potential $[\mathrm{mV}]$ \\
\hline WTF & $67.2 \pm 0.8$ & $-34.89 \pm 2.27$ \\
LRF & $65.7 \pm 0.7^{\text {n.s. }}$ & $-36.79 \pm 6.19^{\text {n.s. }}$ \\
CON & $60.9 \pm 1.0$ & $-59.84 \pm 10.27$ \\
FN & $59.8 \pm 0.9^{\text {n.s. }}$ & $-58.01 \pm 10.13^{\text {n.s. }}$ \\
\hline
\end{tabular}

Data is shown in the form: mean $\pm \mathrm{SD}$. Three different samples in each group were used to determine the contact angle and $\zeta$ potential of the group.

n.s.: No significant differences were detected between the WTF and LRF substrates, and between the CON and FN substrates by the two-sided Student's t-test. 


\section{Figure captions}

\section{Figure 1}

Scanning electron micrographs of WTF ( $a$ and b) and LRF (c and d) films (A), photographs of chondrocytes grown on WTF (e), LRF (f), CON (g), and FN (h) substrates at $24 \mathrm{~h}$ after seeding (B), and chondrocytes grown on LRF substrate with soluble GRGDSP peptides in the serum-free medium at concentrations of 0 (i), 0.1 (j), 1 (k), and 10 (l) $\mu \mathrm{g} / \mathrm{ml}(\mathrm{C})$. Scale bar = $500 \mu \mathrm{m}$ (a and c), $200 \mathrm{~nm}$ (b and d), and $200 \mu \mathrm{m}$ (e-1).

\section{Figure 2}

Temporal changes in adhesive force to detach a single chondrocyte on WTF and LRF substrates (A), and CON and FN substrates (B). Curve fitting was done using a smoothing spline regression. Data is shown in the form: mean \pm SD. Asterisks indicate significant differences between the WTF and LRF groups, and sharps indicate significant differences between the CON and FN groups $(*: \mathrm{p}<0.05$, **, \#\#: $\mathrm{p}<0.01$, ***: $\mathrm{p}<0.001$; two-sided Student's t-test).

\section{Figure 3}

Time-dependent changes in spreading area of a single chondrocyte grown on WTF and LRF substrates (A), and CON and FN substrates (B). Curve fitting was done using a smoothing spline regression. Data is shown in the form: mean \pm SD. Sharps indicate significant differences between the CON and FN groups (\#: $p<0.05$, \#\#: $p<0.01$; two-sided Student's t-test).

\section{Figure 4}

Immunofluorescence staining of F-actin (A, C, E, G, I, K, M, and O) and vinculin (B, D, F, H, $\mathrm{J}, \mathrm{L}, \mathrm{N}$, and P) in a chondrocyte grown on WTF (A-D), LRF (E-H), CON (I-L), and FN (M-P) substrates at 6 (A, B, E, F, I, J, M, and N) and 12 (C, D, G, H, K, L, O, and P) h after seeding. Scale bar $=20 \mu \mathrm{m}$.

\section{Figure 5}

Temporal changes in relative mRNA expression levels of integrins $\alpha 5$ (A) and $\beta 1$ (B), aggrecan (C), and collagen type II (D) in chondrocytes grown on WTF, LRF, CON, and FN substrates normalized to the expression level at baseline $(0 \mathrm{~h})$. Curve fitting was done using a smoothing spline regression. Data is shown in the form: mean \pm SD. Asterisks indicate significant differences between the WTF and LRF groups (*: $\mathrm{p}<0.05$; two-sided Student's by t-test).

Figure 6 
Scanning electron micrographs of WTF ( $a$ and b) and LRF (c and d) sponges (A), and photographs of regenerated cartilage tissues using WTF (e, g, i, k, m, and o) and LRF (f, h, j, l, $\mathrm{n}$, and p) sponges at $3(\mathrm{e}, \mathrm{f}, \mathrm{i}, \mathrm{j}, \mathrm{m}$, and $\mathrm{n}$ ) and $7(\mathrm{~g}, \mathrm{~h}, \mathrm{k}, \mathrm{l}, \mathrm{o}$, and $\mathrm{p}$ ) days in culture (B). Panels e-h are safranin-O stained, panels i-l are collagen type II immunostained, and panels m-p are collagen type I immunostained. Scale bar $=500 \mu \mathrm{m}$ (a and c) and $200 \mu \mathrm{m}(\mathrm{b}, \mathrm{d}$, and e-p). 


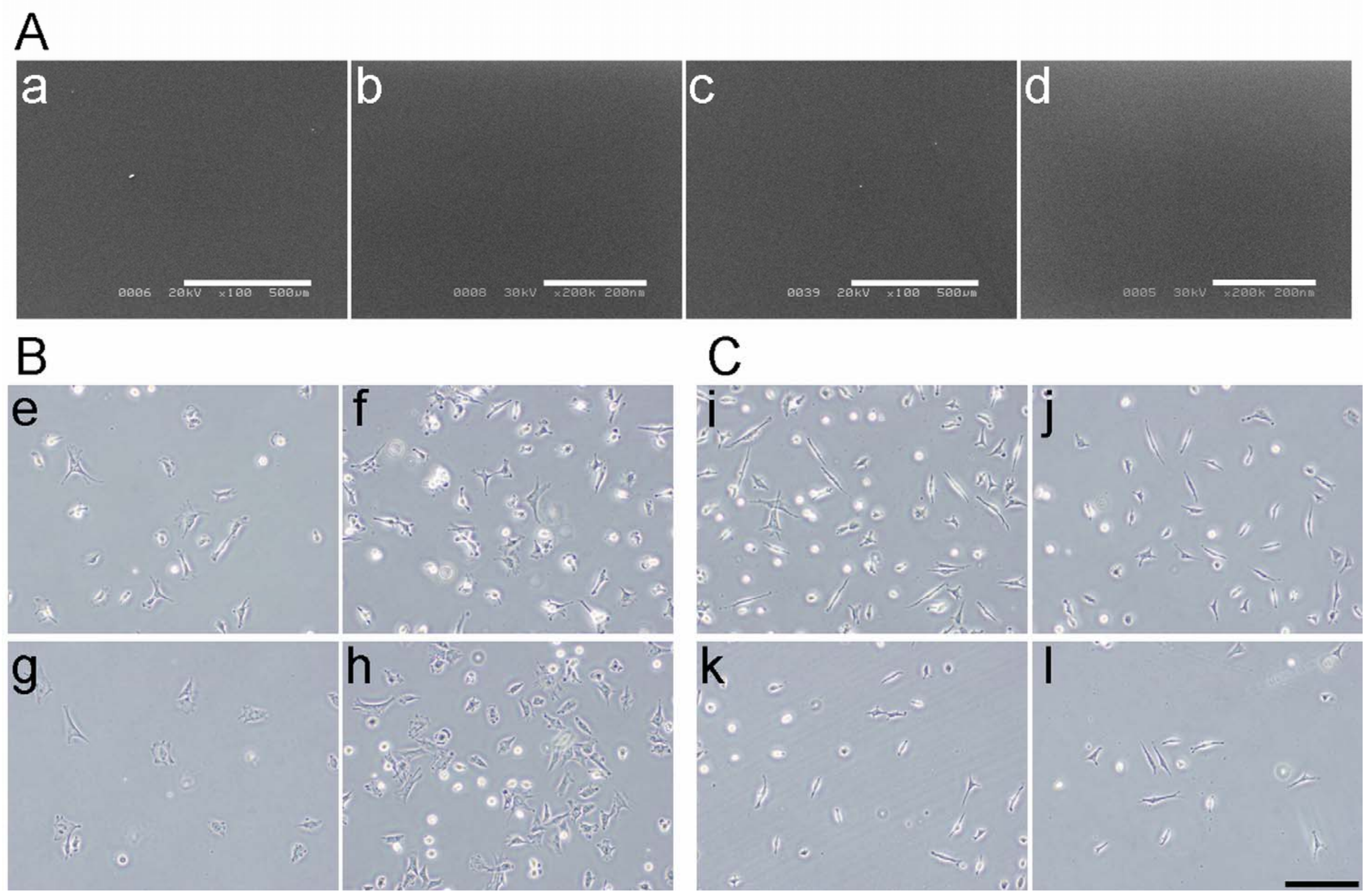




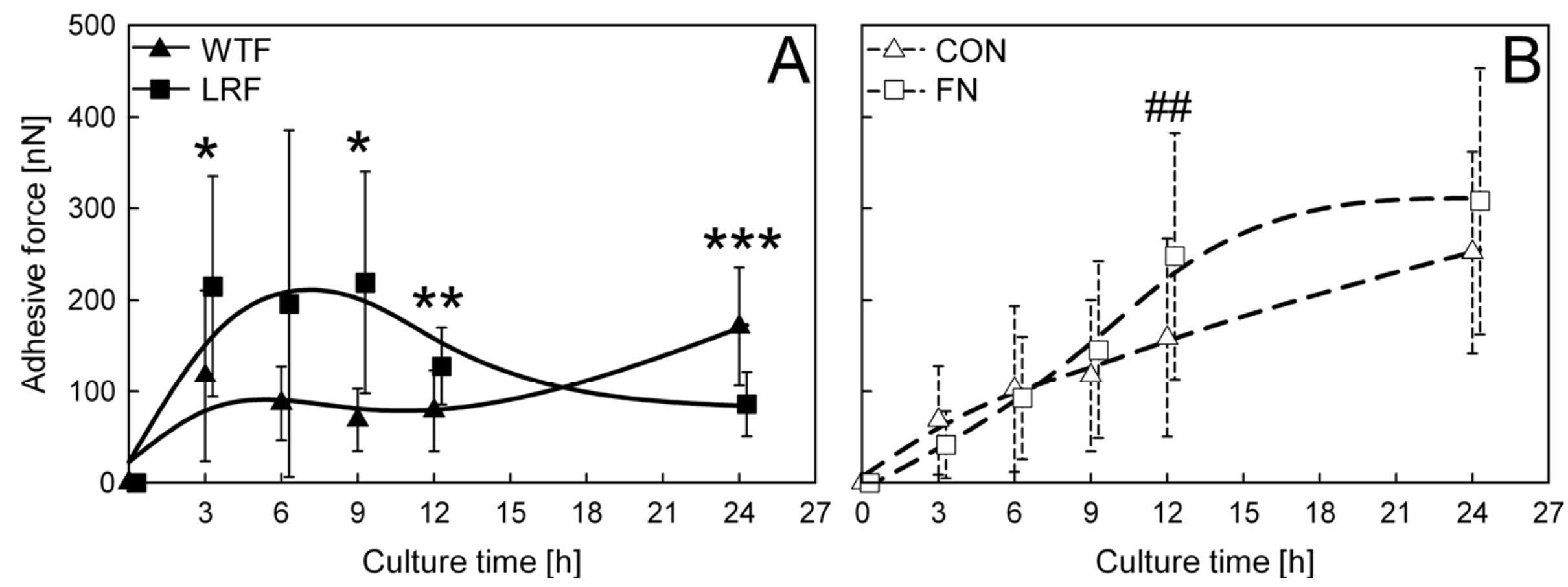



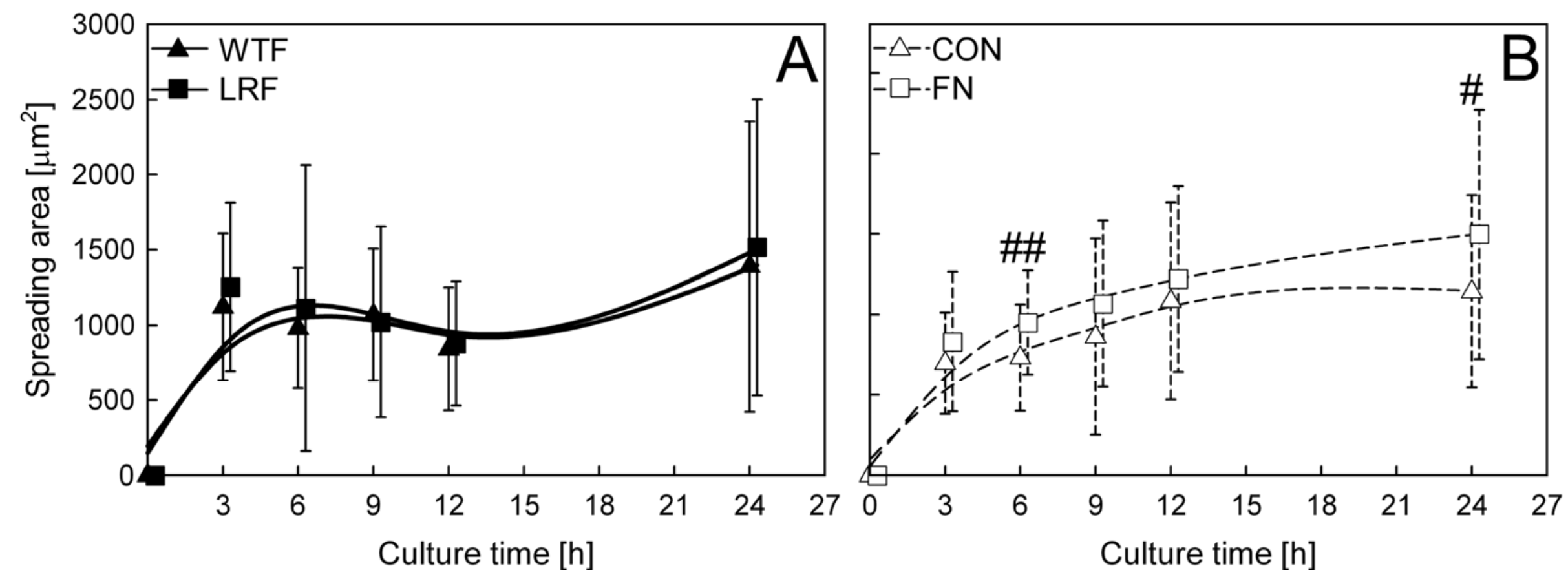
$6 \mathrm{~h}$

$12 \mathrm{~h}$

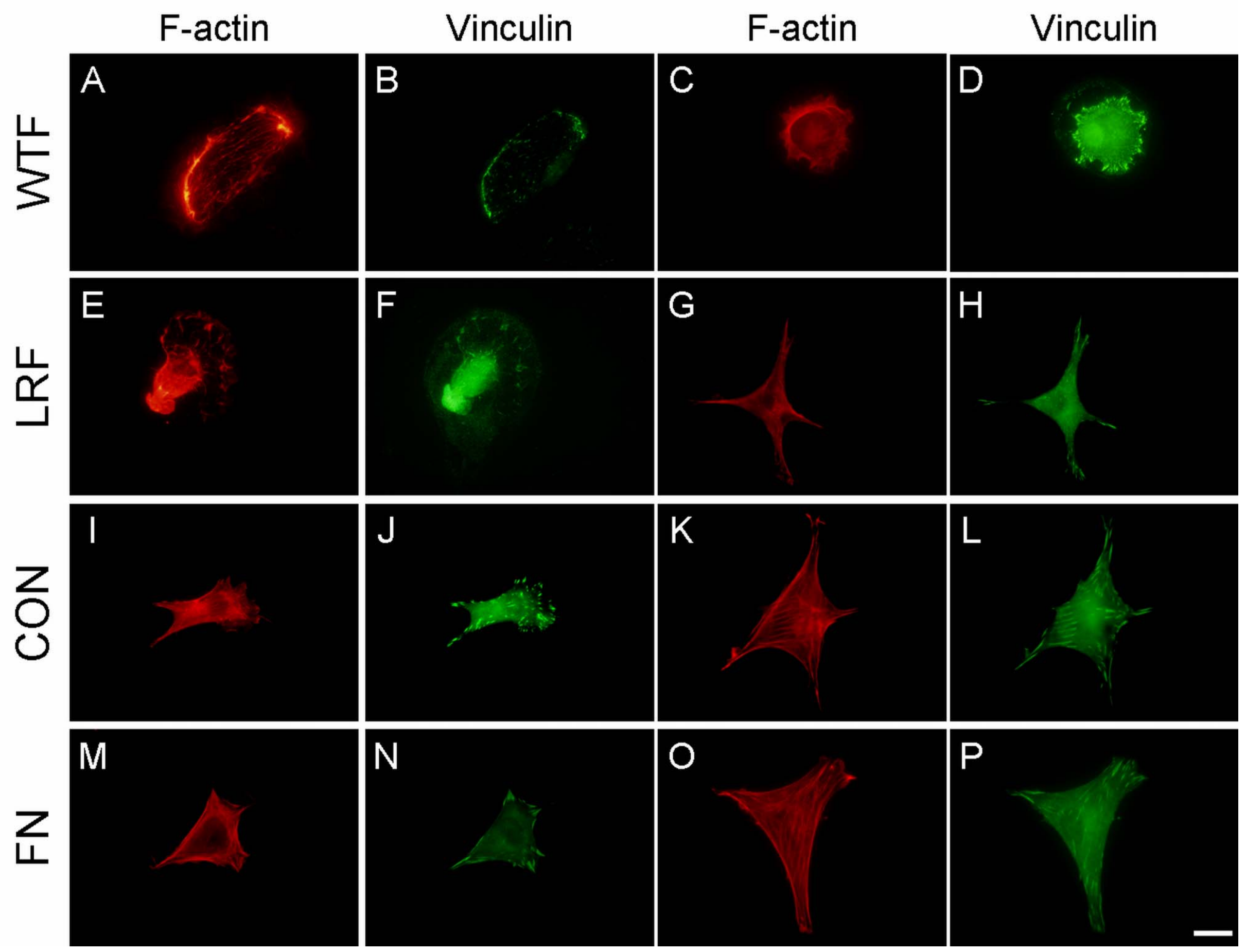



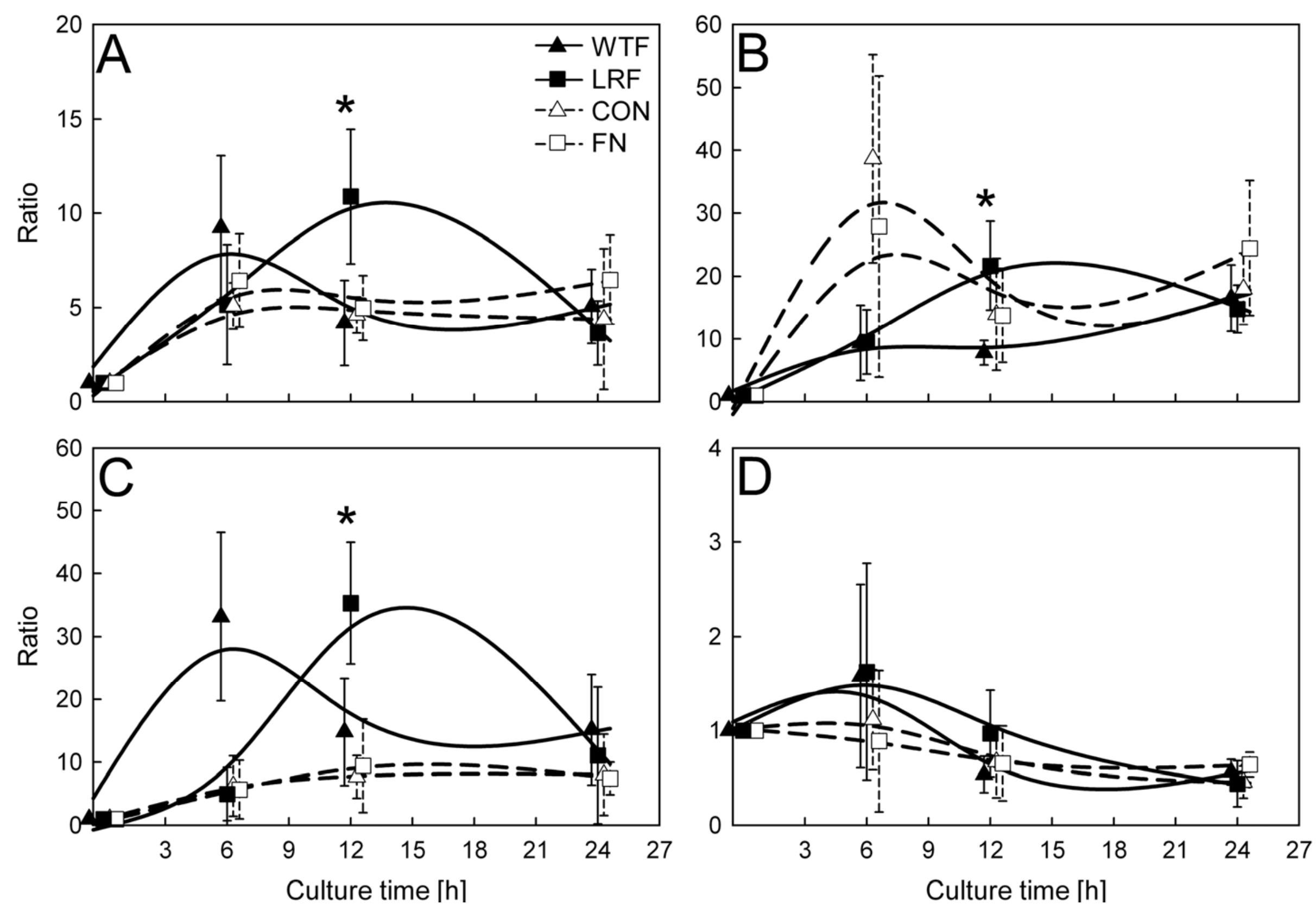
A

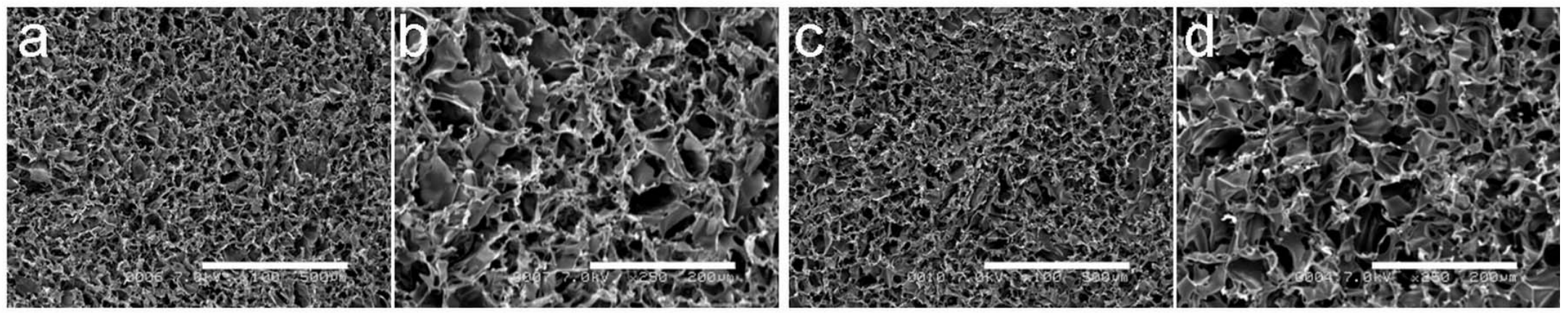

B

3 days

7 days

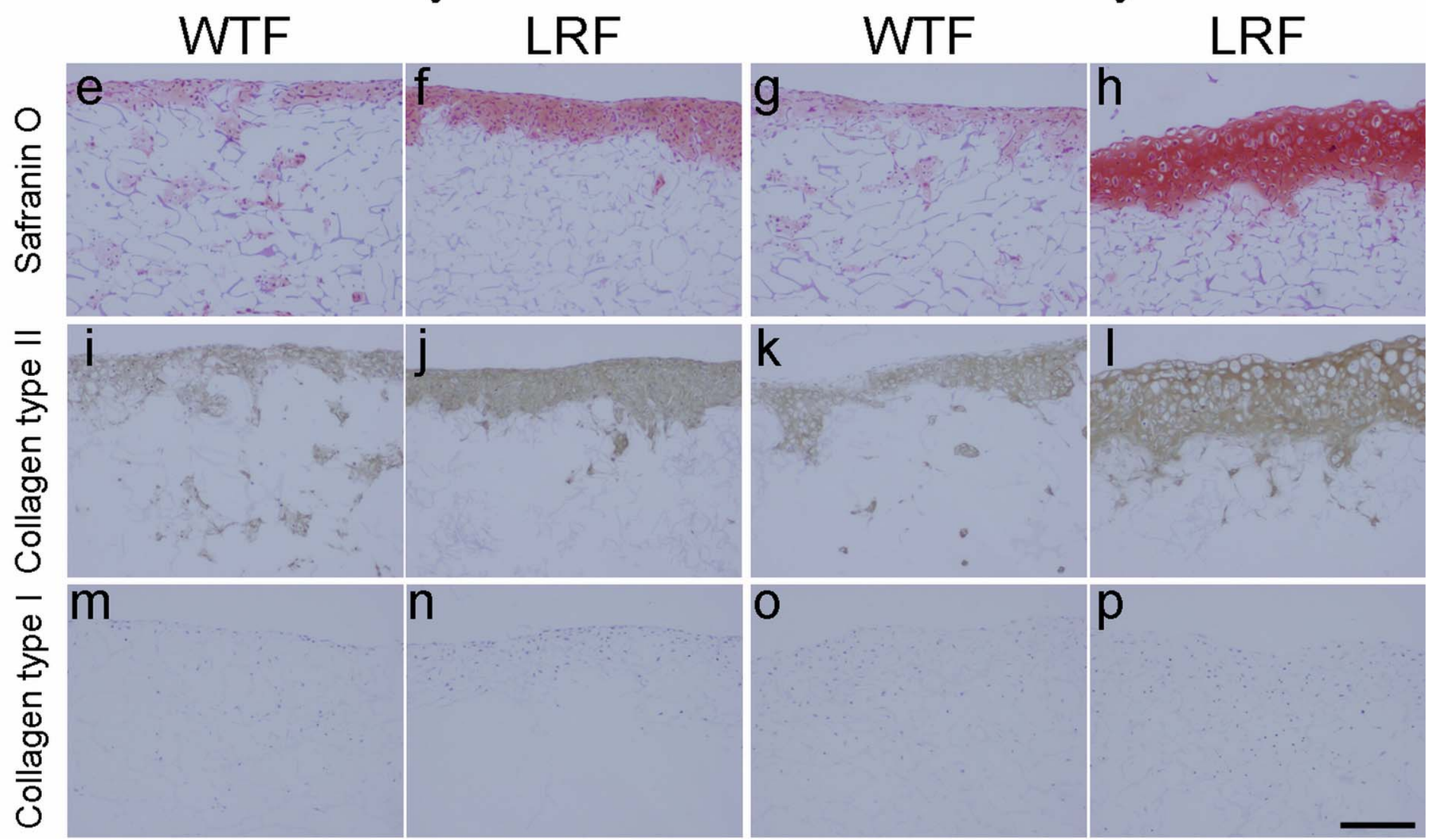

